\title{
L'interculturel comme paradigme de transgression par rapport au culturalisme
}

Martine PRETCEILLE, Professeur Emérite des Universités

"Un honnête homme, c'est un homme mêlé » Montaigne, Essais III, Chapitre IX, De la vanité

Au moment où la diversité culturelle est reconnue comme un élément structurant et non plus contextuel de nos sociétés, apparait ainsi un premier paradoxe, car il faut admettre dans le même temps que la culture n'est plus une notion opérationnelle pour appréhender cette diversité. Il nous faut donc accepter de déconstruire le concept de culture au profit de l'interculturalité. Malgré les acquis de la recherche, au-delà des mots et des usages souvent inflationnistes, l'interculturel reste peu investi par les sciences humaines et sociales. On observe un phénomène de résistance proche de l'idéologie où la force des convictions domine par rapport aux démonstrations. Ainsi, le paradigme interculturel qui est une forme de transgression par rapport au paradigme culturaliste se heurte à des résistances voire à des opérations de dénigrement qui nous interdisent de penser le divers d'aujourd'hui. En effet, le terme « interculturel » sert souvent à recouvrir n'importe quelle action (sociale ou éducative), n'importe quelle recherche pourvu que ces dernières fassent référence, de manière plus ou moins claire, à la culture.

J'ai dénoncé depuis ma thèse cette dérive et ce danger qui bloquent toute progression théorique et pratique. Bien que de plus en plus usité, le terme perd, paradoxalement, de sa force et de sa pertinence. Il convient donc de préciser, d'une part, en quoi l'interculturel est un paradigme qui permet de penser pour penser le divers et une autre vision du monde, et d'autre part, de s'interroger sur les résistances et les confusions par rapport à la prégnance du culturalisme. En effet, un paradigme n'est pas une simple description d'une grande famille de phénomènes mais déjà une interprétation, une manière de traiter, une conception d'ensemble, une philosophie voire une éthique.

En quoi l'interculturel peut-il être considéré comme un nouveau paradigme ?Si nous reprenons la définition de Thomas S. Kuhn, qui considère qu'un paradigme «fournit à une communauté de chercheurs des problèmes types et des solutions » (11) et qu'il constitue un ensemble de connaissances généralement admises à une époque donnée par la communauté scientifique et formant un corpus théorique, on doit reconnaître que le paradigme interculturel permet de déterminer des faits significatifs, d'établir une concordance entre les faits et une 
théorie. L'établissement d'un paradigme permet à une communauté scientifique de choisir des problèmes dont on suppose qu'ils ont une solution dans le cadre dudit paradigme.

Entre le «zéro culturel », c'est-à-dire l'ignorance ou la négation de la dimension culturelle et le «tout culturel », c'est-à-dire une survalorisation de la culture comme facteur déterminant les conduites, la marge de manœuvre est étroite. Ainsi, la reconnaissance, relativement récente, des cultures tend à une «dictature»du culturel par réduction de l'individu à son appartenance culturelle et par une survalorisation de la dimension culturelle qui débouchent sur une dérive culturaliste et différentialiste. De ce fait, on peut considérer que le paradigme interculturel est transgressif par rapport au paradigme précédent, notamment celui du culturalisme.

La référence à la culture, aux cultures ne suffit pas, il convient d'expliciter à quelle théorie générale de la culture on se réfère et sur quelle conception de l'être humain et de la société on se fonde. Les connaissances ne peuvent se limiter à une simple description sur le registre photographique de l'expérience mais nécessitent une théorie qu'il ne faut pas confondre avec une quelconque forme d'idéologie et de dogmatisme. La perspective interculturelle correspond à cette tentative à partir d'un ensemble conceptuel et méthodologique.

Si la reconnaissance de la diversité culturelle fait l'objet d'un consensus large et spontané, encore faut-il mettre en lumière les principes philosophiques, sociologiques et anthropologiques qui la sous-tendent. Les années soixante-dix ont vu l'émergence des vocables comme différence et pluralisme. Aujourd'hui, on évoque davantage les termes de diversité culturelle et d'interculturel. Comment faut-il comprendre et interpréter ce changement ? Notre postulat est que le passage de la différence à la diversité, du pluralisme à la pluralité, du multiculturalisme à l'interculturalité ne relève pas d'une simple évolution sémantique mais suggère un changement profond de perspective. De fait, tout changement de paradigme correspond à des transformations dans la vision du monde (156).

En effet, l'interculturalité est en convergence avec les philosophies de la différence même si nous contestons ce terme, au profit de celui de diversité - de Jacques Derrida, Gilles Deleuze, Michel Foucault, Michel de Certeau, Jean-François Lyotard et F. Jacques, ainsi que les philosophies de l'altérité avec Labarrière, Paul Ricoeur, Emmanuel Lévinas et celle la pensée du complexe vulgarisée notamment par Edgar Morin, mais aussi les pensées de la variation de Isabelle Stengers, Henri Atlan, Michel Serres et Albert Jacquard, notamment. Cet environnement conceptuel confirme que le paradigme interculturel n'est pas une transgression veine et de type aléatoire et assure ses prémisses. Il renvoie, d'une part, à un 
courant théorique et épistémologique profond en émergence et en construction, d'autre part à un cadrage théorique renouvelé afin de répondre à des problèmes de terrain qui eux-mêmes déterminent de nouveaux objets d'étude.

\section{Points d'ancrage notionnels du paradigme interculturel}

Toujours selon Kuhn, « la découverte d'un paradigme commence avec la conscience d'une anomalie, c'est-à-dire l'impression que la nature, d'une manière ou d'une autre, contredit les résultats attendus dans le cadre du paradigme qui gouverne la science normale » (83). En effet, le paradigme culturaliste structuré à partir des notions de différence, de reconnaissance de cultures distinctes, identifiées et identifiables, d' «identité-racine », de logique unitaire et homogène ne permet pas de rendre compte du processus d'hétérogénéisation de plus en plus important et de plus en plus rapide des sociétés et des groupes.

\section{I.1. Diversité, multiplicité, dynamiques}

L’idée de diversité, à l'opposé de celle de différence, repose moins sur la reconnaissance de catégories culturelles que sur celle de dynamiques et de processus du «mixte». En effet, toute culture est « une œuvre ouverte» susceptible d'interprétations et d'expressions plurielles. Cette réfraction à l'infini des cultures à travers le temps et les lieux, les conjonctures et les subjectivités, les structures et les circonstances pose la question de la pertinence du concept de culture dans un monde marqué par une complexité croissante. Le paradigme interculturel permet de travailler les transgressions opérées par les acteurs qui construisent leur identité culturelle en innovant, en franchissant les frontières, en s'autorisant à développer les marges et à développer une «pensée de la trace par opposition à une pensée de système » (Glissant, « Conférence », 1993).

Depuis la fin des années soixante-dix, des tentatives se multiplient pour proposer un cadrage théorique qui permette de penser le divers et la pluralité. En effet, les notions de culture, identité, traits culturels, culture d'appartenance, catégorisation et identification sont insuffisantes pour rendre compte des multi-appartenances, des mutations, des individualisations, des personnalisations des comportements et des conduites, des transgressions, des zébrures, des parcours alternatifs, de la marginalité et des brassages.

Il a déjà été largement démontré, par le biais des études interculturelles notamment, que les notions d'identité et de culture ne sont définissables que dans un cadre intersubjectif et sont le produit de relations et d'interactions. Il est en de même pour des notions comme le lignage, les rituels, les codes, les structures, etc. Mohder Kilani a mis à jour le caractère 
fictionnel et discursif du lignage dont le sens n'est pas préconstruit mais au contraire tributaire des interprétations que les membres d'une communauté échangent: «L'organisation lignagère apparaît comme une pure forme qui procède essentiellement par captation, arrangement, déplacement et exclusion, et jamais par recours à un contenu primordial et intangible » (225). Le lignage ne serait donc pas un élément stable mais un événement, une dynamique, une construction exprimée à travers le langage mais aussi les comportements et les conduites des acteurs.

Cette opération de déconstruction/reconstruction des concepts «traditionnels » de l'ethnologie a aussi été engagée par Jean-Loup Amselle et Elikia M'Bokolo qui, dans une étude consacrée à l'ethnie et au tribalisme en Afrique, montrent parfaitement leur caractère discursif et pragmatique dans la mesure où elles ne renvoient pas à des réalités stables mais à des constructions liées aux interactions entretenues entre les membres d'un même espace social et géographique. La mise en question de la notion d' « ethnie » entraîne dans son sillage la mise en question des notions comme celle de lignage, mais aussi de clan, de tribu, etc. : «Toutes ces notions redoublent l'idéologie de la société dont elles sont extraites, ne sont en fait que des «formes symboliques » permettant la réunion de certains effectifs humains sous la bannière d'une communauté imaginaire de sang ou de race, et cela notamment dans le cadre des Etats » (36).

En abandonnant le paradigme de la culture et des aires culturelles, ce sont les fractures, les écarts, les métissages, les chemins de traverse qui sont mis en évidence et travaillés car porteurs de sens. Arjun Appadurai propose de «penser la configuration des formes culturelles comme fondamentalement fractales, c'est-à-dire comme dépourvues de frontières, de structures ou de régularités euclidiennes » (85) tout en les combinant avec leurs chevauchements et leurs ressemblances. Le paradigme interculturel fait ainsi partie de ces voies qui tentent de penser l'hybride, le segmentaire et l'hétérogénéité devenus la norme.

Il nous faut donc reconnaître la porosité et les hybridations entre les systèmes, les structures, les individus et les groupes, car chacun transgresse de plus en plus souvent et de plus en plus facilement les références de son ou de ses propres groupes d'appartenance. Serge Gruzinski voit dans l'hétérogénéité des processus de construction de l'identité, la diversité et l'actualité de la «pensée métisse $»^{1}:$ «Chaque sujet est doté d'une série d'identités, ou des références plus ou moins stables, qu'il active simultanément ou successivement [...]. L'identité est une histoire personnelle, qui est à son tour liée à des capacités différentes de l'intériorisation ou au déni des normes inculquées » (53). 


\section{I.2. Priorité aux interactions et à la relation}

L'affaiblissement pour ne pas dire la fin des paradigmes de causalité, de cohérence et de logique unitaire et homogène ouvre la voie à la notion de réseau et au primat de la notion de relation au détriment de l'esprit de système et de structure. La démarche interculturelle, confondue souvent avec une approche culturelle voire multiculturelle, met l'accent sur les processus et les interactions qui unissent et définissent les individus et les groupes les uns par rapport aux autres. Il ne s'agit plus de s'arrêter sur les caractéristiques auto-attribuées ou hétéro-attribuées de chacun. La priorité donnée à la relation sur les entités se retrouve chez Edouard Glissant quand il évoque la pensée de l'errance qui se dégage de « la déstructure des compacités nationales, hier encore triomphantes, et en même temps, des naissances difficiles et incertaines aux formes nouvelles d'identité » (Poétique, 30). Plus qu'une variable supplémentaire, la culture donne ou redonne une place au sujet, aux interactions, au contexte, aux processus d'analyse en miroir,

Dans le même temps, la compréhension d'autrui exige un travail sur soi afin d'éviter de sombrer dans une forme de tautologie expérientielle où on reproduit, consciemment ou non, du même, c'est-à-dire du soi. L'interrogation identitaire de soi par rapport à autrui fait partie intégrante de la démarche interculturelle.

\section{I.3. La culturalité}

À tort ou à raison, à bon ou à mauvais escient, nous sommes sans cesse contraints d'utiliser des images qui remplacent la réalité vécue et observée, images sans lesquelles nous ne pourrions ni décrire une situation qui n'est pas devant nous, ni réfléchir dessus mais qui deviennent parfois de véritables faisceaux de conceptions sur le monde. Le concept de culture tel qu'il est utilisé aujourd'hui nous cache la complexité du monde, notamment tous les processus de créolisation et de bigarrure. Il fonctionne, comme l'a si bien dit Claude LéviStrauss, comme un concept opératoire qui permet de parler des cultures mais qui ne correspond à aucune réalité.

Dans un contexte marqué par la diversité et la diversification, les cultures ne peuvent plus être appréhendées comme des entités indépendantes, hors de toute forme d'actualisation dans le social, le politique et le communicationnel. On a, en effet, moins à faire à des entités culturelles stables qu'à des fragments. Le temps n'est plus ni aux nomenclatures, ni aux monades mais au contraire aux bigarrures et aux transgressions, car chaque individu a la possibilité de s'exprimer et d'agir en s'appuyant non seulement sur ses codes d'appartenance, mais aussi sur des codes de référence librement choisis ou encore assimilés. La culture, comme la langue, est un lieu de mise en scène de soi et des autres et les individus 
sélectionnent les traits culturels selon leurs intérêts, les contraintes de la situation et les enjeux, symboliques ou non d'ailleurs. Ce sont donc les fragments culturels qui deviennent significatifs et non plus les cultures dans leur globalité.

Dans la mesure où la culture et les cultures sont de plus en plus l'objet de manipulations, de bricolages, de transformations provisoires ou définitives, intentionnelles ou inconscientes, individuelles ou collectives, on aura perçu la vanité de chercher à les classer, les nommer ou encore à les catégoriser d'autant que ce ne sont pas des actes neutres. En effet, pour Pierre Bourdieu, nommer c'est classer. De même pour Lyotard, nommer c'est prendre perspective : «la définition nominale est une désignation, mais la désignation, bien loin d'être une adéquation du signe à la chose, est, comme la perspective..., une 'décision' qui fait exister d'un coup, ensemble, le signe et sa référence » (51).

Par ailleurs, du fait que les cultures sont de plus en plus mouvantes, alvéolaires, tigrées, labiles, que ce sont les traces culturelles qui sont efficientes et non pas les structures, il convient de proposer une notion susceptible d'en rendre compte, car le concept de culture reste trop marqué par une approche descriptive, globalisante et statique. L'individu est rarement en contact avec le «tout» de sa culture, encore moins de la culture de l'Autre; tout au plus est-il confronté à un «précipité ». C'est en ce sens que la question centrale est moins celle d'une initiation aux cultures que la compréhension des processus culturels dans leur valeur d'engendrement des comportements et des discours. Pour toutes ces raisons, nous proposons la notion de culturalité qui, comme l'adjectif sociétal en sociologie, focalise sur les processus, les dynamiques qui traversent les groupes et les sociétés. Elle permet d'appréhender les transformations, les manipulations, les dynamiques.

\section{I.4. L'altérité}

L'interculturel comme paradigme induit un renversement de perspective qui transgresse notre vision de la culture, des cultures pour s'approcher de celle de l'altérité. Si la reconnaissance de la diversité culturelle n'est pas le retour des cultures et du pluralisme culturel, elle signifie, par contre, le retour de l'altérité. Travailler sur les cultures, c'est nécessairement retrouver les philosophies de l'altérité. C'est cette irruption de l'altérité qui exige une mise en perspective plurielle et fluide, car autrui ne se laisse pas voir, ne se laisse pas regarder et maîtriser facilement. L'Autre n'est pas un objet, mais une aventure, un devenir, un événement. L'anthropologie est, en ce sens, arrimée à l'éthique. L'initiation aux cultures, l'apprentissage des cultures, quelle que soit la finesse des savoirs, reste extérieure à la rencontre de l'Autre, car elle s'appuie sur un discours de catégorisation et d'attribution à partir notamment de savoirs factuels et descriptifs. Les connaissances abstraites et 
globalisantes des cultures oblitèrent la reconnaissance de l'individu singulier, elles fonctionnent comme un filtre voire un écran et produisent ainsi un artefact qui justifie en retour les analyses culturalistes.

Toute focalisation excessive sur les spécificités d'autrui conduit à l'exotisme, à une fossilisation des pratiques culturelles avec pour conséquence une accentuation, consciente ou non, des stéréotypes et des préjugés. Apprendre à voir, à écouter, à être attentif à autrui, apprendre la vigilance et l'ouverture dans une perspective de diversité et non de différences renvoient à la reconnaissance et à l'expérience de l'altérité, expérience qui s'acquiert et se travaille dans la durée. Autrui ne se laisse pas saisir en dehors d'une communication et d'un échange. Les singularités vulgarisées à tort sous le vocable de différences sont plus directement perceptibles que l'universalité qui nécessite analyse. En ce sens, parler de reconnaissance et d'acceptation des différences, c'est faire l'économie d'une réflexion dont l'objet est notamment de rapprocher ces singularités d'une universalité sous-jacente. La tension singularité/universalité est au cœur de la démarche interculturelle.

\section{Des résistances voire des obstacles à la diffusion du paradigme interculturel}

Ce nouveau paradigme reste, paradoxalement, ignoré voire combattu au profit d'un recours systématique à une ethnographie différentialiste confondue souvent avec une de ses dérives à savoir le culturalisme, par hypertrophie de la variable culturelle. On assiste en quelque sorte à des formes de résistance particulièrement bien étudiées par Kuhn qui pointe le fait que « les scientifiques n'ont pas pour but, normalement, d'inventer de nouvelle théorie, et ils sont souvent intolérants envers celles qu'inventent les autres »(47). De fait, la science possède son propre conservatisme. Les scientifiques peuvent rester attachés à l'ancien paradigme pour diverses raisons : refus de changement de vision du monde, attachement au paradigme auquel on doit ses principales réussites, conformité aux attentes notamment des appels d'offre, etc. Par ailleurs, on voit aussi un certain nombre de scientifiques se détacher progressivement du paradigme dominant (sans pour autant toujours adhérer à un nouveau paradigme).

Si l'expression «interculturelle »'est pas nécessairement reprise, on notera avec force la proximité de pensée et on se félicitera de l'émergence d'un courant qui, s'il n'est pas encore reconnu, n'en est pas moins représentatif d'une cohérence en émergence : «Le fait même qu'une nouveauté scientifique importante émerge si souvent simultanément de plusieurs laboratoires, prouve d'une part la nature fortement traditionnelle de la science 
normale et d'autre part le fait que cette entreprise traditionnelle prépare parfaitement la voie de son propre changement $\gg(99)$.

Les résistances proviennent aussi du poids des options politiques et de leur traduction dans des pratiques sociales et éducatives voire dans les recherches. En effet, penser la diversité culturelle à partir de catégories et des caractéristiques culturelles permet de justifier le développement des politiques de recrutement sur des bases de plus en plus ethniques et la recherche d'une adéquation entre les origines des personnels et les publics avec lesquels ils doivent travailler.

Cette crispation sur une logique de frontières et d'appartenance conduit à une forme de radicalisation de la différence. Toute réduction des phénomènes culturels à la connaissance des cultures risque non seulement d'être caduque mais de développer des situations d'enfermement réciproque. La focalisation sur les singularités, le découpage du réel et sa réduction à un conglomérat d'unités autorisent malheureusement les politiques de rejet et d'exclusion et permettent de faire l'impasse sur une philosophie et une éthique communes.

\section{Un cadre d'analyse}

\section{1. Une anthropologie générative}

L'anthropologie se définit comme une réflexion sur l'être humain et sa diversité selon le principe de variation. Elle correspond à une démarche qui consiste à partir du particulier pour construire une théorie de l'humain. Le concept central n'est pas comme on le pense trop souvent celui de culture mais celui d'altérité. On confond souvent l'anthropologie avec l'ethnologie qui, elle, s'intéresse avant tout à l'être humain dans sa globalité et dans ses différences. Entre la connaissance des différences culturelles (approche ethnographique) et la compréhension de la variation culturelle (approche anthropologique), il n'y a pas qu'un changement de formulation, mais également le passage d'une analyse en termes de structures et d'états à celle de processus, de situations complexes, imprévisibles et aléatoires, en un mot à une science du divers.

C'est essentiellement à une anthropologie des problèmes qu'il conviendra de recourir plus qu'à une ethnologie descriptive et adjectivante. Georges Balandier évoque une science sociale générative qui oriente vers des interprétations définies en termes d'action et d'interactions complexes, en termes d'engendrement. Par anthropologie générative, on entendra une connaissance, non pas des caractéristiques, mais des phénomènes et des 
processus culturels : acculturation, assimilation, résistance culturelle, identité, métissage, etc., dans leur dimension générique.

La description est aux antipodes de la compréhension et par voie de conséquence de la résolution des difficultés. Elle part d'un point, elle est linéaire alors que les événements sont de l'ordre du global et du multidimensionnel. Dès lors, il convient d'apprendre à discerner l'essentiel de l'accidentel, l'universalité des processus de la singularité de leur actualisation. Ce type d'analyse représente une alternative par rapport à des sciences humaines encore trop imprégnées de l'analogie avec la nature.

\section{III.2. La pragmatique}

À une période centrée sur les structuralismes, les nomenclatures, les typologies et les fonctionnalismes succède une ère marquée par les problématiques, les mutations, les chemins de traverse, les fractures. Dans la mesure où l'accent est moins porté sur la structure, mais sur le sujet qui agit et donc interagit, on est dans le domaine de la pragmatique et du fonctionnement instrumental de la culture, par opposition à sa valeur de détermination et de modelage. Toute culture, loin d'être une donnée, « est le produit de négociations continuelles avec le monde extérieur, négociations à travers lesquelles s'affirme un horizon, une identité qu'on ne peut que définir que comme une création continue » (Schnapper 151). Il ne s'agit pas de rechercher d'hypothétiques réalités culturelles mais, au contraire, d'appréhender une forme de pragmatique culturelle, de comprendre comme se construit le culturel dans des situations complexes. Marqués par le contexte, inscrits dans un réseau d'intersubjectivités, les faits culturels sont utilisés pour signifier quelque chose, pour dire et pour agir. Ils assurent, en ce sens, une fonction pragmatique au détriment des fonctions structurantes, dénotatives dans lesquelles on a souvent tendance à enfermer la culture.

En conséquence, il s'agit de favoriser l'émergence d'une aptitude à cerner les ajustements culturels, les traces, dans un souci, non pas d'adaptation et de conformité mais de renouvellement permanent. On s'attardera moins sur la culture comme déterminant les comportements mais sur la manière dont l'individu utilise les traits culturels pour dire et se dire, pour s'exprimer verbalement, corporellement, socialement, personnellement. Or, les signes culturels sont polysémiques et le sens ne peut être donné qu'à partir d'une analyse et non pas par un simple recours à un répertoire sémiotique.

Prendre en compte la diversité culturelle comme dynamique, c'est rompre avec les analyses et les pratiques qui s'appuient sur la description des différences pour expliquer les comportements et les attitudes par une appartenance à un groupe culturel. Il ne s'agit pas de s'appuyer sur des techniques de codage et de décodage des signes culturels mais de tendre à 
comprendre autrui et non à le décrire et à le stigmatiser. De fait, la compréhension des situations ne relève pas d'un paradigme du savoir (inventaire de particularismes, par exemple) mais d'une compétence pragmatique qui permet de saisir la culture à travers le langage, la communication, les actes, les comportements, c'est-à-dire de comprendre «une culture en acte », d'une « culture mise en scène » par opposition à une « culture-système ».

Les pratiques, attitudes et comportements culturels sont donc approchés via un vécu subjectif, en tant que symptôme dans la relation que l'individu entretient avec l'environnement. La sociologie compréhensive qui tente de répondre à «une hétérogénéisation du monde » (Maffesoli 25) en prônant « une compréhension systémique au spectre étendu », revalorise la signification pour l'individu de ses comportements au-delà de leur objectivité, au-delà de leur déterminisme de de leur groupe d'appartenance ou de culture. Il s'agit en fait de chercher à «préserver la singularité des actes et des situations face au rouleau compresseur du positivisme » (63).

\section{3. L'interculturel est une herméneutique}

Aucun fait n'est d'emblée «interculturel » et la qualité d' «interculturel » n'est pas un attribut de l'objet. Seule l'analyse interculturelle peut lui conférer ce caractère. C'est le regard qui crée l'objet et non l'inverse. Par exemple, dire que nous sommes dans une société interculturelle et que nous travaillons sur des objets interculturels est, en fait, un abus de langage. La spécificité de l'approche interculturelle réside bien dans le mode d'interrogation et non dans un champ d'application présenté comme interculturel, encore moins comme un objectif. Erigé à partir d'un dualisme entre une construction théorique, méthodologique et épistémologique, et une réalité d'expérience, l'interculturel relève de la compréhension et de l'action. L'étroite imbrication de ces deux registres lui confère un statut oscillant entre un herméneutisme et un humanisme au sens où «l'humanisme n'est pas, comme on le croit trop souvent, une simple définition morale de l'homme et de sa capacité à établir un régime raisonnable entre les contemporains d'une même époque »(Duvignaud 156).

L'ancrage de l'anthropologie dans la phénoménologie conduit à penser la rencontre de l'Autre non pas comme le produit de connaissances mais comme une re-connaissance. À une ethnologie centrée sur les différences et la distinction, il sera privilégié une anthropologie relationnelle et herméneutique, c'est-à-dire un travail d'interprétation et d'analyse des interactions, des inter-définitions des individus et des groupes. L'interculturel ne se situe pas du côté d'une « vérité d'adéquation qui serait celle de l'algorithme », c'est-à-dire d'une suite de raisonnements qui fournirait une solution, mais d'une «vérité par transparence, recoupement et reprise » pour reprendre une distinction opérée par Maurice Merleau-Ponty 
(84). L'enjeu consiste à sortir du modèle d'explication pour passer à une compréhension du changement. Celui-ci ne peut être perçu à partir des caractéristiques individuelles et groupales, mais à partir de problématiques centrées sur les contacts, les interactions, les acculturations, les dysfonctionnements, problématiques associées par ailleurs à une obligation d'interprétation renouvelée et permanente. L'analyse ne peut pas porter sur la nature des interlocuteurs, mais uniquement sur la manière dont ils se donnent à voir, dont ils se mettent en scène (voir Goffman, 1956), sur leur présentation. Elle reste au niveau des apparences et non de l'essence (voir Maffesoli). La recherche de transparence (culturelle, notamment) s'oppose à tout travail sur de l'individuel et donc sur du complexe, de l'aléatoire et du polysémique. L'approche interculturelle, qui n'a pas de caractère prédictif, permet de comprendre et de modéliser des situations complexes à partir d'un mode d'intelligibilité. Elle est en ce sens, une herméneutique.

Personne n'a accès au «tout» de la culture d'autrui, mais seulement à une connaissance partielle et ponctuelle, elle-même dépendante du contexte et de la «mise en scène » des acteurs. Chacun doit alors s'appuyer sur une « connaissance graduée d'éléments significatifs » (Schutz 219). Apprendre à distinguer, dans une situation donnée, les éléments qui relèvent de ce que certains appellent une spécificité culturelle de ceux qui sont l'expression d'une individualité propre, tel est l'enjeu de l'approche interculturelle. Pour reprendre les propos d'Alfred Schutz, nous dirons que « le modèle culturel du groupe n'est pas un lieu de refuge mais un champ d'aventure, pas une évidence, mais un point d'interrogation à investiguer, pas un instrument utile pour clarifier les situations embrouillées mais une situation problématique et même difficile à maîtriser » (232).

C'est, en quelque sorte, la culture en acte, et non la culture comme objet qui est au cœur du paradigme interculturel. Si la culture et les cultures ne sont pas des données mais des constructions, la compétence correspondante sera davantage de l'ordre des savoir-faire que des savoirs, ceux-ci étant réduits au rang d'accessoires puisqu'ils n'ont qu'une valeur illustrative et non démonstrative. La compétence culturelle s'inscrit dans une connaissance du multiple et non de la totalité ou de l'homogène. Elle exige une capacité à penser en termes de conjectures et d'hypothèses. La connaissance culturelle ne saurait être réduite à une combinatoire, même fine et complexe, une géométrisation, une mécanique d'éléments culturels. L'approche par la connaissance des cultures peut suffire tant que les interlocuteurs se conduisent selon les normes et les modèles répertoriés. Les difficultés commencent dès que les uns ou les autres n'entrent pas, pour une raison ou une autre, dans le cadre attendu, car le formé n'est pas nécessairement le prototype de son groupe. En ce sens, les 
connaissances culturelles n'améliorent pas nécessairement, ni la rencontre, ni la relation, ni la communication.

L'anthropologie est en réalité une herméneutique dans la mesure où elle effectue un travail d'interprétation et non d'explication. En conséquence, c'est une formation à l'analyse et non une démarche descriptive qui fonde la découverte et la compréhension de la diversité culturelle. Le point nodal réside dans l'élaboration d'une pensée duale et non pas dualiste, d'une pensée plurielle et non pas pluraliste.

\section{Bibliographie}

Amselle, Jean-Loup et Elikia M'Bokolo. Au cæur de l'ethnie. Ethnie, tribalisme et Etat en Afrique. Paris : La Découverte, 1999.

Appadurai, Arjun. Après le colonialisme. Les conséquences culturelles de la globalisation. Paris : Payot, 2001 (1996).

Balandier, Georges. Anthropo-logiques. Paris : Librairie générale française, 1985.

Barber, Bernard. «Resistance by Scientists to Scientific Discovery". Sciences CXXXIV (1961) : 596-602.

Duvignaud, Jean. Introduction à la sociologie. Paris : Gallimard, 1966.

Glissant,Edouard. «Conférence inaugurale du Carrefour des littératures européennes, Strasbourg, 4 novembre 1993 ».Le monde, 5 novembre 1993.

---. Poétique de la relation. Paris : Gallimard, 1990.

Gruzinski, Serge. La pensée métisse. Paris, Éditions Fayard, 1999.

Kilani, Mondher. L'invention de l'autre. Essais sur le discours anthropologique. Lausanne: Payot, 1994.

Kuhn, Thomas S. La structure des révolutions scientifiques. Paris: Flammarion, 1983 (Or, 1962).

Lyotard, Jean-François. Rudiments païens. Paris : Union Générale des Editions, 1977.

Maffessoli, Michel. La connaissance ordinaire. Précis de sociologie compréhensive. Paris : Méridiens, 1985.

Merleau-Ponty, Maurice. La prose du monde. Paris : Gallimard, 1969.

Montaigne, Michel. Essais II, Chap. IX, De la vanité, 1588. Paris : Gallimard, 1965.

Schnapper, Dominique. « Modernité et acculturations ». Communications 43 (1986).

Schutz, Alfred, Le chercheur et le quotidien, phénoménologie des sciences sociales. Paris : Méridiens / Klinksieck, 1987 (Or, 1971). 


\section{NOTE}

${ }^{1}$ La notion de pensée métisse est contestée voire contestable car elle suppose la reconnaissance d'entités distinctes bien identifiées qui font l'objet d'un processus du métissage. (cf. Alexis Nouss). 